CYBERNETICS AND INFORMATION TECHNOLOGIES • Volume 15, No 7

Special Issue on Information Fusion

Sofia $\bullet 2015$

Print ISSN: 1311-9702; Online ISSN: 1314-4081

DOI: $10.1515 /$ cait-2015-0092

\title{
The Impact of the Quality Assessment of Optimal Assignment for Data Association in a Multitarget Tracking Context
}

\author{
J. Dezert ${ }^{1}$, A. Tchamova ${ }^{2}$, P. Konstantinova ${ }^{3}$ \\ ${ }^{1}$ The French Aerospace Lab, Chemin de la Huniere, F-91761 Palaiseau, France \\ ${ }^{2}$ Institute of Informations and Communication Technologies, BAS, 1113 Sofia, Bulgaria \\ ${ }^{3}$ European Polytechnical University, Pernik, Bulgaria \\ Emails: jean.dezert@onera.fr tchamova@bas.bg pavlina.konstantinova@gmail.com
}

Abstract: The main purpose of this paper is to apply and to test the performance of a new method, based on belief functions, proposed by Dezert et al. in order to evaluate the quality of the individual association pairings provided in the optimal data association solution for improving the performances of multisensormultitarget tracking systems. The advantages of its implementation in an illustrative realistic surveillance context, when some of the association decisions are unreliable and doubtful and lead to potentially critical mistake, are discussed. A comparison with the results obtained on the base of Generalized Data Association is made.

Keywords: Data association, Belief Functions, PCR6 fusion rule, multitarget tracking.

\section{Introduction}

The problem of Data Association (DA) is central in modern multi-target tracking (MTT) systems' design $[1,2]$. It is related to the process of associating uncertain measurements (observations) to known tracks and is conditioned and motivated by the most important function of each surveillance system - to keep and to improve target tracks maintenance performance. In the monosensor context it corresponds to proper sensor observations partitioning (at a given scan) to the predicted states of the targets in such a way, that their tracks' updates to be as precise, correct and reasonable, as much as possible.

There are several approaches developed to resolve the correlation ambiguities and to select the best observation-track pairings, based on different models. Some of them establish a reward matrix based on Kinematic only Data Association 
(KDA) and on a probabilistic framework [3,4]. Some of them rely on Belief Functions (BF) [5-9] and motivate the incorporation of the advanced concepts for Generalized Data Association (GDA) [6-8], allowing the introduction of a target attribute (target type, radar cross section, etc.) into the association logic, in order to improve the track maintenance performance in complicated situations (closely spaced/crossing targets), when kinematics data are insufficient for coherent decision making. The main characteristics consists in applying Dezert-Smarandache theory (DSmT) of plausible and paradoxical reasoning [8] to model and to process the utilized attribute data. In the most common case, when the surveillance system provides only information kinematic (such as range, azimuth, elevation), obtained during a given scan, the most common way of dealing is to solve the optimal DA solution and to use all solutions (pairings) to update tracks, even if some of the parings have poor quality. It could yields in fact, a bad/wrong track updating, and, as a result, the overall tracking performance could be degraded substantially.

The most recent method proposed by Dezert \& Benameur [10] to evaluate the Quality Assessment of Data Association (QADA) encountered in multiple target tracking applications in a mono-criterion context, and recently extended in [11] for the multi-criteria context, deal just with the case above given. It assumes that the rewards matrix is known and has been obtained by a method chosen by the user. It is based on belief functions for establishing the quality of pairings (interpreted as a confidence score) belonging to the optimal data assignment solution based on its consistency (stability) with respect to all the second best solutions, provided by a chosen algorithm.

The main purpose of our paper is to serve as a preliminary study of MTT performance evaluation based on QADA-KDA approach, and to discuss its advantages in an illustrative multi-target tracking scenario. We will make also comparison between its performance and the results obtained on the base of GDA.

The paper is organized as follows. Section 2 describes the problem of DA in multitarget tracking context. Section 3 provides details about the new method [10] for quality assessment of an optimal DA solution. In Section 4 the simulation scenario and results are presented and discussed. A conclusion is given in Section 5.

\section{Data association problem in multitarget tracking context}

Data Association is a very important and most decisive step in the multitarget tracking surveillance process. The DA problem consists in finding the global optimal assignment of the targets $T_{i}, i=1, \ldots, m$, to some measurements $z_{j}$, $j=1, \ldots, n$, at a given time $k$ by maximizing the overall gain in such a way that no more than one target is assigned to a measurement, and reciprocally.

The so called $m \times n$ rewards (gain/payoff) matrix $\Omega=[\omega(i, j)]$ is defined by the elements $\omega(i, j)>0$, representing the gain of association of the target $T_{i}$ with the measurement $z_{j}$. These elements are usually homogeneous to the likelihood ratios. In some cases $\omega(i, j)>0$ represent the normalized distances between the 
measurement $j$ and target $i$, and in this case DA problem consists in finding the best assignment, minimizing the overall cost.

The goal of the optimal assignment problem is to find a $m \times n$ binary association matrix $A=[a(i, j)]$, where

$$
a(i, j)=\left\{\begin{array}{lc}
1 & \text { if measurement } z_{j} \text { is assigned to track } T_{i}, \\
0 & \text { otherwise. }
\end{array}\right.
$$

The association matrix maximizes the global reward $R(\Omega, A)$, given by:

$$
R(\Omega, A) \stackrel{\Delta}{=} \sum_{i=1}^{m} \sum_{j=1}^{n} \omega(i, j) a(i, j) .
$$

The importance of the assignment problem is quite clear and various successful solutions to its solving already exist. Among the well known are KuhnMunkres algorithm (known as Hungarian) $[12,13]$ and its extension proposed by Bourgeois and Lassalle in [14] to rectangular matrices. More sophisticated Murty's method [15] provides not only the first best assignment, but also the $m$-best assignments in order of increasing the cost, as shown in the examples of [10,11].

The best optimal assignment solution is not necessarily unique, as well as the second best one. Usually in MTT algorithms the first best assignment solution is taken as a hard decision for association. But in some real practical cases of dense multi-target and cluttered environment, DA problem is difficult to solve, because some of the associations decisions $a(i, j)$ are unreliable and doubtful, so they could lead to potentially critical mistakes. For example, in case of incorrect determination of the incoming measurements for two tracks in such a way, that they are too close, the solution of the assignment problem, that is the core of GNN, is impossible to be sufficiently explicit. In such a case, it will be more cautious not to rely on all the pairings confirmed in the first best solution, but only on some of them which are trustable enough, according to the apriori defined threshold level. Utilizing the already obtained and available $m$-best assignments solutions, Dezert et al. $[10,11]$ provide a very efficient method for achieving this important knowledge.

\section{Quality assessment of the optimal DA}

The first and the second best assignment matrices $A_{1}$ and $A_{2}$ are used [10], in order to establish the quality of the specific associations (pairings), satisfying the condition $a_{1}(i, j)=1$. The main idea behind QADA method is to compare the values $a_{1}(i, j)$ in $A_{1}$ with the corresponding ones $a_{2}(i, j)$ in $A_{2}$ and to identify the change (if any) of the optimal pairing $(i, j)$. In our MTT context, $(i, j)$ means that the measurement $z_{j}$ is associated with the target $T_{i}$. A quality indicator is established, depending on both the stability of the pairing and its relative impact on the global reward. The proposed method works also when the 1st and 2nd-best optimal assignments $A_{1}$ and $A_{2}$ are not unique, i.e., there are multiplicities 
available. The construction of the quality indicators is based on BF theory and Proportional Conflict Redistribution fusion rule No 6 (PCR6), defined within DSm theory [8].

It depends on the type of the pairing matching in the way, described below:

- In case when $a_{1}(i, j)=a_{2}(i, j)=0$, one has a full agreement on the "nonassociation" of the given pairing $(i, j)$ in $A_{1}$ and $A_{2}$. This "non-association" has no impact on the global reward values $R_{1}\left(\Omega, A_{1}\right)$ and $R_{2}\left(\Omega, A_{2}\right)$, so it will be useless to utilize it in DA. Hence, the quality indicator value is set to $q(i, j)=0$.

- In case when $a_{1}(i, j)=a_{2}(i, j)=1$, one has a full agreement on the "association" of the given pairing $(i, j)$ in $A_{1}$ and $A_{2}$. This "association" has different impacts on the global reward values $R_{1}\left(\Omega, A_{1}\right)$ and $R_{2}\left(\Omega, A_{2}\right)$. In order to estimate the quality of this matching association, one establishes two basic belief assignments (bba), $m_{S}(),. s=1,2$, according to both sources of information ( $A_{1}$ and $A_{2}$ ). The frame of the discernment, that one reasons on, consists of a single hypothesis $X=\left(T_{i}, z_{j}\right)$ : measurement $z_{j}$ belongs to $\operatorname{track} T_{i}$. The ignorance is modelled by the proposition $X \cup \bar{X}$, where $\bar{X}$ is a negation of hypothesis $X$,

$$
\left\{\begin{array}{l}
m_{s}(X)=a_{s}(i, j) . \omega(i, j) / R_{s}\left(\Omega, A_{s}\right), \\
m_{s}(X \cup \bar{X})=1-m_{s}(X) .
\end{array}\right.
$$

Applying the conjunctive rule of combination $m_{1}(.) \otimes m_{2}($.$) one gets:$

$$
\left\{\begin{array}{l}
m_{12}(X)=m_{1}(X) m_{2}(X)+m_{1}(X) m_{2}(X \cup \bar{X})+m_{1}(X \cup \bar{X}) m_{2}(X), \\
m_{12}(X \cup \bar{X})=m_{1}(X \cup \bar{X}) m_{2}(X \cup \bar{X})
\end{array}\right.
$$

The pignistic transformation [15] is applied in order to obtain pignistic probabilities, built on the basis of fused basic belief assignments, such as $\operatorname{Bet} P(X)=m_{12}(X)+\frac{1}{2} \cdot m_{12}(X \cup \bar{X})$ and $\operatorname{Bet} P(\bar{X})=\frac{1}{2} \cdot m_{12}(X \cup \bar{X})$. Then one chooses the quality indicator about the association $\left(T_{i}, z_{j}\right)$ as $q(i, j)=\operatorname{Bet} P(X)$.

- In case when $a_{1}(i, j)=1$ and $a_{2}(i, j)=0$, then a disagreement (conflict) on the association $\left(T_{i}, z_{j}\right)$ in $A_{1}$ and $A_{2}$ is detected. One could find the association $\left(T_{i}, z_{j_{2}}\right)$ in $A_{2}$, where $j_{2}$ is the measurement index, such that $a_{2}\left(i, j_{2}\right)=1$. In order to define the quality of such conflicting association $\left(T_{i}, z_{j}\right)$, one establishes two basic belief assignments (bba), $m_{S}().(s=1,2)$ according to both sources of information ( $A_{1}$ and $A_{2}$ ). The frame of discernment, one reasons on, consists of the following two propositions: $X=\left(T_{i}, z_{j}\right)$, and $Y=\left(T_{i}, z_{j_{2}}\right)$. The ignorance is modelled by the proposition $X \cup Y$. Then one obtains: 


$$
\begin{gathered}
\left\{\begin{array}{l}
m_{1}(X)=a_{1}(i, j) \cdot \omega(i, j) / R_{1}\left(\Omega, A_{1}\right), \\
m_{1}(X \cup Y)=1-m_{1}(X) ;
\end{array}\right. \\
\left\{\begin{array}{l}
m_{2}(Y)=a_{2}\left(i, j_{2}\right) \cdot \omega\left(i, j_{2}\right) / R_{2}\left(\Omega, A_{2}\right), \\
m_{2}(X \cup Y)=1-m_{2}(Y) .
\end{array}\right.
\end{gathered}
$$

Different rules of combination (Dempster-Shafer's, Dubois-Prade's, Yager's [16] could be chosen to work with a normalized combined BBA. The method [10] recommends to use the Proportional Conflict Redistribution rule No 6 (PCR6), proposed originally in DSmT framework [8], because it has been proved very efficient in practice. With PCR6, the following fusion result $m_{\text {PCR6 }}()=.m_{1}(.) \otimes m_{2}($.$) is obtained:$

$$
\left\{\begin{array}{l}
m_{\mathrm{PCR} 6}(X)=m_{1}(X) m_{2}(X \cup Y)+m_{1}(X) \cdot \frac{m_{1}(X) \cdot m_{2}(Y)}{m_{1}(X)+m_{2}(Y)}, \\
m_{\mathrm{PCR} 6}(Y)=m_{1}(X \cup Y) m_{2}(Y)+m_{1}(Y) \cdot \frac{m_{1}(X) \cdot m_{2}(Y)}{m_{1}(X)+m_{2}(Y)}, \\
m_{\mathrm{PCR} 6}(X \cup Y)=m_{1}(X \cup Y) m_{2}(X \cup Y) .
\end{array}\right.
$$

The decision is taken on the base of the pignistic transformation:

$$
\begin{aligned}
& \operatorname{Bet} P(X)=m_{\mathrm{PCR} 6}(X)+\frac{1}{2} \cdot m_{\mathrm{PCR} 6}(X \cup Y), \\
& \operatorname{Bet} P(Y)=m_{\mathrm{PCR} 6}(Y)+\frac{1}{2} \cdot m_{\mathrm{PCR} 6}(X \cup Y) .
\end{aligned}
$$

The quality indicators are chosen as: $q(i, j)=\operatorname{Bet} P(X)$ and $q\left(i, j_{2}\right)=\operatorname{Bet} P(Y)$. The absolute quality factor becomes:

$$
Q_{\mathrm{abs}}\left(A_{1}, A_{2}\right)=\sum_{i=1}^{m} \sum_{i=1}^{n} a_{1}(i, j) \cdot q(i, j)
$$

\section{Simulation scenario and results}

The noise-free multitarget tracking simulation scenario (Fig. 1) consists of three air targets moving in parallel from west to east at a constant velocity of $100 \mathrm{~m} / \mathrm{s}$ and a distance between them $150 \mathrm{~m}$. The stationary sensor is located at the origin. The sampling period is $T_{\text {scan }}=5 \mathrm{~s}$ and the measurement standard deviations are $0.5 \mathrm{deg}$ and $65 \mathrm{~m}$ for azimuth and range respectively. The surveillance of moving targets is performed during 15 scans. Fig. 2 shows the respective noised scenario. 


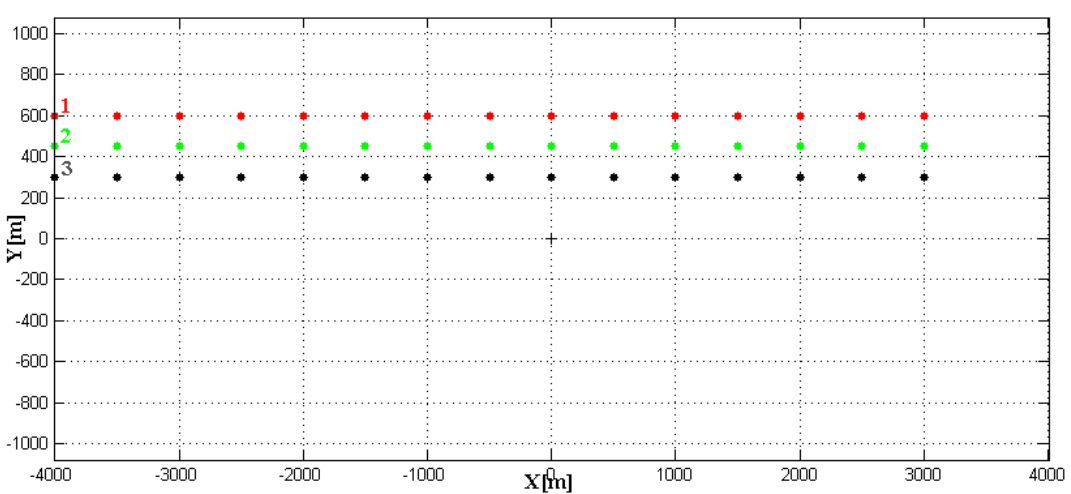

Fig. 1. Noise-free MTT scenario

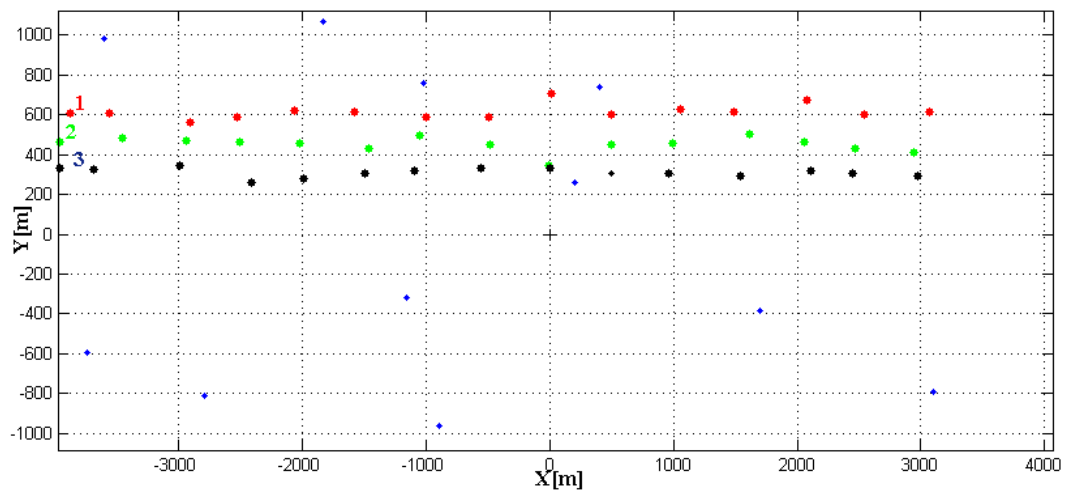

Fig. 2. Noised MTT scenario

The classical target tracking algorithm was run, consisting of two basic steps: (i) data association to associate the proper measurements (distance, angle) with correct targets and (ii) track filtering to update the targets state vectors, once the optimal assignment was found. In our simulation the Converted Measurement Kalman Filter [1] is used.

In this work we will focus our attention on DA step, which is a very important, and the most decisive one in multitarget tracking. The Global Nearest Neighbour (GNN) [1] approach is used in order to make a decision for data association. One obtains the assignment matrix $\operatorname{AMat}(i, j), i=1, \ldots, m, j=1, \ldots, n$, based on the normalized distances between measurement $j$ and target $i$. In order to eliminate unlikely (kinematics-based) observation-to-track pairings, the classical validation test is carried on the Mahalanobis distance $[1,2] \quad d^{2}(i, j)$ computed from the measurement $z_{j}(k)$ at a given time moment $k$, and its prediction $\hat{z}_{i}(k / k-1)$ :

$$
d^{2}(i, j) \stackrel{\Delta}{=}\left(z_{j}(k)-\hat{z}_{i}(k / k-1)\right)^{\prime} S^{-1}(k)\left(z_{j}(k)-\hat{z}_{i}(k / k-1)\right) \leq \gamma .
$$


Assuming the given measurement vector's size to be $M$, the quantity $d_{i j}^{2}$ could be interpreted as a sum of the squares of $M$ independent Gaussian random variables with zero means and unit standard deviations. For this reason $d_{i j}^{2}$ have $\chi_{M}^{2}$ distributions with $M$ degrees of freedom and allowable probability of a valid observation falling outside the gate. In our case, a probability of $1 \%$ is approved, then from the table of the chi-square distribution [2] one obtains the threshold $\gamma=9.21$. In fact, this value represents the biggest possible distance value associated with the observation-to-track pairings. Based on this, one assumes that if $j$-th measurement does not fall in the gate of target $i$, then the value, associated with this pairing $(i, j)$ in the assignment matrix could be set to be big enough (in our case it equals 100), in order to prepare the assignment matrix for the next step. The classical Munkres and Katta-Murty methods [15] are used in order to obtain the first and the second best assignment solutions for measurement-to-track associations. By minimizing the sum of the chosen pairings' distances, a binary association matrix $A=[a(i, j)]$ is obtained. Fig. 3 shows the typical MTT performance, based on the classical GNN approach with Kinematic only DA (KDA), when one does not utilize additional procedures to improve the quality of DA.

In case of noised measurements, it is evident that at scan number 9, tracks 2 and 3 change their directions, becoming crossing, instead of following their parallel moving behavior. It is because of incorrect determination of the incoming measurements in such a way, that they are too close and the solution of the assignment problem, which is the core of GNN, is impossible to be categorical. The problem consists also in the proximity of the targets (inter-distance of $150 \mathrm{~m}$ ), and in the bad sensor distance resolution of $\sigma_{\mathrm{D}}=65 \mathrm{~m}$. It leads to a wrong GNN association decision.

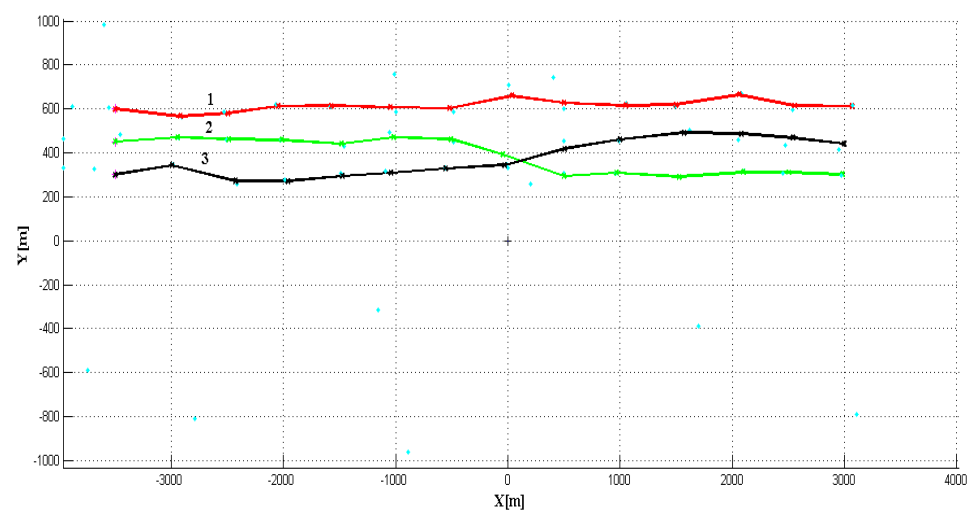

Fig. 3. Typical MTT performance with KDA

A criterion for a minimal admissible measurements' distances is chosen here as $d_{\min }<\sigma_{\mathrm{D}} / 2$. During scan No 9, one has: $d_{23}<d_{\min }$. 
In such a critical case, one needs to utilize some additional information in order to avoid associations' miscorrelations. Here the method in [10] is applied. The goal is to estimate the quality of the questionable pairings $\left(T_{2}, z_{2}\right)$ and $\left(T_{3}, z_{3}\right)$. One obtains the corresponding reward matrix $\Omega=[\omega(i, j)]$ with elements $\omega(i, j)$, representing the gain of the associations of target $T_{i}, i=1, \ldots, m$, with measurement $z_{j}, j=1, \ldots, n$, It is achieved as $\Omega=[\omega(i, j)]=10-\operatorname{AMat}(i, j)$. The reason for this expression relates to the already determined maximal normalized distance $\gamma=9.21$ according to the table of chi-square distribution. The data association deals with finding the global optimal assignment of the targets to some measurements by maximizing the overall gain in such a way, that no more than one target is assigned to a measurement, and reciprocally. This is an equivalent measure for optimality, since it is the global minimum of the distances.

The algorithm, based on [10] was automatically applied during scan No 9, because the minimum distance between observations No 2 and No 3 is under the accepted limits $d(2,3)=15.83 \mathrm{~m}<\sigma_{\mathrm{D}} / 2$. The quality matrix (Table 1 ) at scan No 9 , containing the quality levels associated with the chosen pairings in the first best solution $A_{1}=\left[\begin{array}{lll}1 & 0 & 0 \\ 0 & 1 & 0 \\ 0 & 0 & 1\end{array}\right]\left(\left\{\left(T_{1}, z_{1}\right),\left(T_{2}, z_{2}\right),\left(T_{3}, z_{3}\right)\right\}\right)$ with respect to the second best one $A_{2}=\left[\begin{array}{lll}1 & 0 & 0 \\ 0 & 0 & 1 \\ 0 & 1 & 0\end{array}\right]\left\{\left(T_{1}, z_{1}\right),\left(T_{2}, z_{3}\right),\left(T_{3}, z_{2}\right)\right\}$ is obtained.

Table 1. Quality matrix at scan No 9

\begin{tabular}{|c|c|c|c|}
\hline Obs/Track & 1 & 2 & 3 \\
\hline 1 & 0.773 & 0.000 & 0.000 \\
\hline 2 & 0.000 & 0.504 & 0.000 \\
\hline 3 & 0.000 & 0.000 & 0.498 \\
\hline
\end{tabular}

It is obvious that according to the first best assignment solution, one has: $q\left(T_{1}, z_{1}\right)=0.773 ; q\left(T_{2}, z_{2}\right)=0.504 ; q\left(T_{3}, z_{3}\right)=0.498$. We accept the admissible for a correct association quality threshold to be set to $q T=0.7$.

Based on the associations quality assessment (Table 1) and the accepted quality threshold $q T=0.7$, one could make the following decision: The only pairing, among those, chosen by Munkres algorithm in the first best assignment is $\left(T_{1}, z_{1}\right)$, because its quality level exceeds the accepted reasonable for correct association quality threshold $q\left(T_{1}, z_{1}\right)=0.773>0.7$. Following the decision logic in [10], only $\left(T_{1}, z_{1}\right)$ pairing will be used in the updating process, while the second and the third tracks will keep going under a prediction mode while the next measurements will be available, because $q(2,2)=0.504<0.7$, and 
$q(3,3)=0.498<0.7$. The performance of MTT algorithm, based on the QADA-KDA is shown on Fig. 4.

It is obvious that the reasoned/informed decision taken at scan No 9, based on QADA-KDA method leads to a miscorrelation conflict resolution.

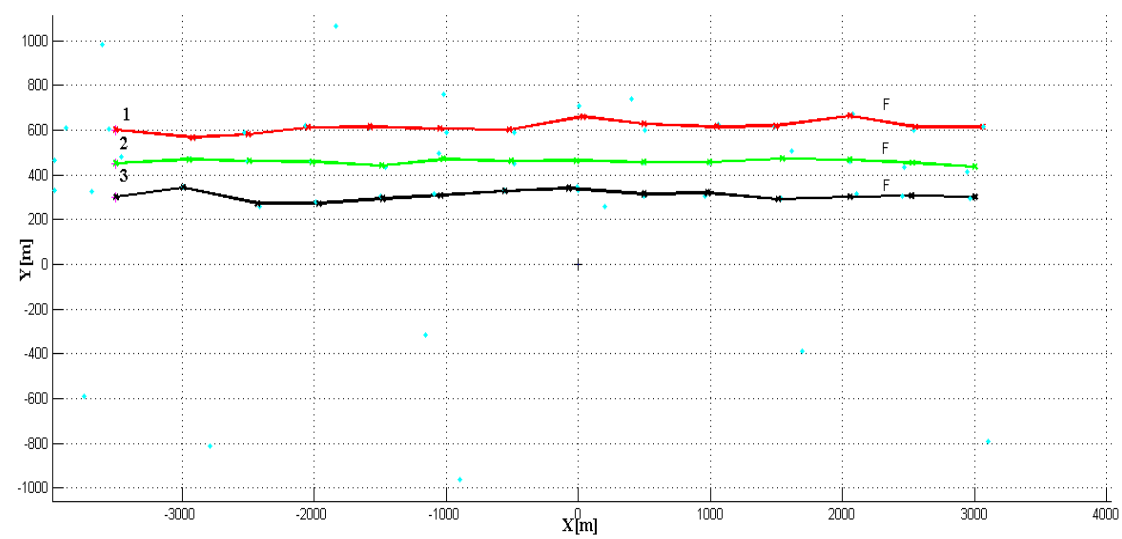

Fig. 4. MTT performance with QADA-KDA

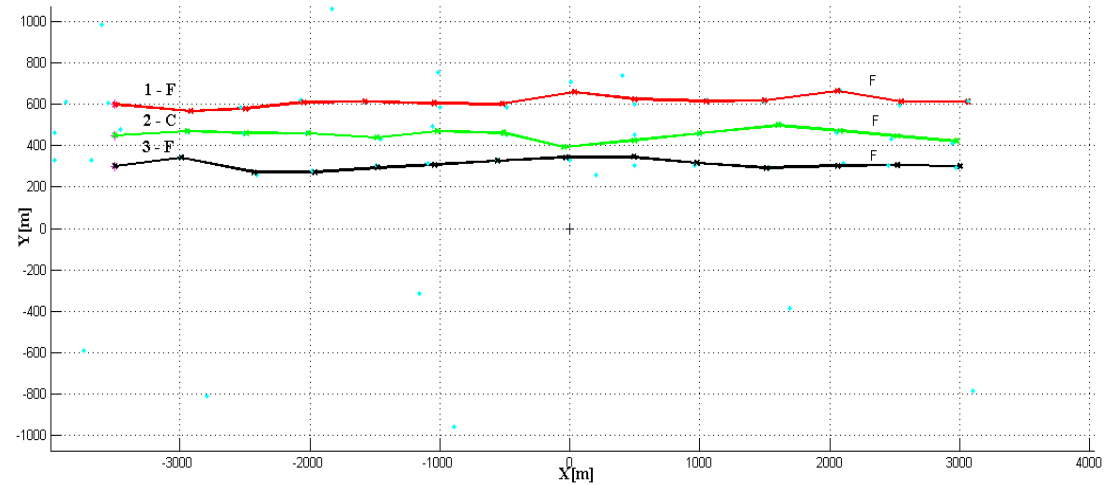

Fig. 5. MTT performance with GDA

In order to compare the obtained by QADA result, a simulation was made by applying GDA (Fig. 5), when the target attribute (target type) is introduced into the association logic, in order to improve the track maintenance performance in the same MTT scenario, with an additional assumption, that targets go from west to east in a group with the following type order (Fighter, Cargo, Fighter .

GDA-MTT improves the process of DA by utilizing target's type decision (based on confusion matrix $C=\left[c_{i j}\right]$ ) coupled with the classical kinematic measurements. The way of constructing the confusion matrix is based on some underlying decision-making process based on specific attribute features measurements. Its elements represent the probability of decisions $T_{\mathrm{d}}$ 


$$
\begin{aligned}
& \left(T_{1} \stackrel{\Delta}{=} \text { Fighter, } T_{2}=\text { Cargo }\right) \text { that the target type is } j \text { when its real type is } i \text { : } \\
& c_{i j}=P\left(T_{d}=T_{j} / \text { TrueTargetType }=T_{i}\right) .
\end{aligned}
$$

In our simulation

$$
C=\left[\begin{array}{ll}
0.95 & 0.05 \\
0.05 & 0.95
\end{array}\right]
$$

GDA is applied at each scan during the whole surveillance process, in order to prevent observation-to-track miscorrelations.

\section{Conclusion}

This work is a preliminary study of MTT performance evaluation based on the new Quality Assessment of the optimal DA method, proposed by Dezert et al. It assures the stability of MTT performance and could be applied in all the cases related to the impossibility of DA to produce an association decision with a high quality. It might concern the cases, when only kinematics measurements are available, as well as the cases when the attribute and kinematic data are both available, because QADA is totally independent of the applied logic to obtain the best DA solution. The work perspective concerns Monte Carlo based evaluation of different, more critical MTT scenarios in a multi-sensor context.

Acknowledgements: This research is supported by AComIn Project "Advanced Computing for Innovation" of IICT-BAS, (2012-2016), Grant 316087, funded by FP7 Capacity Programme (www.iict.bas.bg/acomin/).

\section{References}

1. Y. Bar Shalom, Ed. Multitarget-Multisensor Tracking: Advanced Applications. Artech House, Norwood, 1990.

2. B a r S h a lo m, Y., T. F o r t m a n n. Tracking and Data Association. Academic Press, 1988.

3. B ar Shalom, Y., P. K. Willet, X. Tian. Tracking and Data Fusion: A Handbook of Algorithms. YBS Publishing, Storrs, CT, USA, 2011.

4. He, X., R. Tharmarasa, M. P elletier, T. Kirubaraja nar. Accurate Murty's Algorithm for Multitarget Top Hypothesis. - In: Proc. of Int. Conference of Information Fusion, Chicago, USA, 2011.

5. De ze r t, J., F. S m a r a d d a ch e, A. T ch a mova. On the Blackman's Association Problem. In: Proc. of Int. Conference of Information Fusion, Cairn, Australia, 2003.

6. T cha mova, A., J. De zert, T. S e merddji e v, P. Konstant in ova. Target Tracking with Generalized Data Association Based on the General DSm Rule of Combination. In: Proc. of Int. Conference of Information Fusion, Stockholm, Sweden, 2004.

7. Dezert, J., A. T chamova, T. Semerddjiev, P. Konstantinova. Performance Evaluation of Fusion Rules for Multitarget Tracking in Clutter Based on Generalized Data Association. - In: Proc. of Int. Conference of Information Fusion, Philadelphia, USA, 2005.

8. F. Smarandache, J. Dezert, Ed. Advances and Applications of DSmT for Information Fusion. Vol. 1, 2, 3. American Research Press, 2004, 2006, 2009.

http://www.gallup.unm.edu/ ssmarandache/DSmT.htm 
9. De n o eux, T., N. E1 Zoghby, V. Cherfa ou i, A. Jouglet. Optimal Object Associaton in the Dempster-Shafer Framework. - IEEE Trans. on Cybernetics, 2014 (to appear).

10. D e z e rt, J., K. B e n a m e u r. On the Quality of Optimal Assignment for Data Association. Springer, Lecture Notes in Computer Science, Vol. 8764, 2014, pp. 374-382.

11. De zert, J., K. B en a me ur, L. Ratton, J. F. Grandin. On the Quality Estimation of Optimal Multiple Criteria Data Association Solutions. - In: Proc. of Int. Conference of Information Fusion, Washington, DC, USA, 6-9 July 2015.

12. Ku h n, H. W. The Hungarian Method for the Assignment Problem. - Naval Research Logistic Quarterly, Vol. 2, 1955, pp. 83-97.

13. Munkres, J. Algorithms for the Assignment and Transportation Problems. - Journal of the Society of Industrial and Applied Mathematics, Vol. 5, 1957, No 1, pp. 32-38.

14. B o u r g e o i s, F., J. C. La s s a 11 e. An Extension of the Munkres Algorithm for the Assignment Problem to Rectangular Matrices. - Comm. of the ACM, Vol. 14, 1971, No 12, pp. 802-804.

15. M u rty, K. G. An Algorithm for Ranking All the Assignments in Order of Increasing Cost. Operations Research Journal, Vol. 16, 1968, No 3, pp. 682-687.

16. S m e t s, P., R. K e n n e s. The Transferable Belief Model. - Artificial Intelligence, Vol. 66, 1994, No 2, pp. 191-234. 